\title{
INTEGRATION BY PARTS FOR THE GENERALIZED RIEMANN-STIELTJES INTEGRAL
}

\author{
W. F. PFEFFER
}

(Received 7 July 1981)

Communicated by E. Strzelecki

\begin{abstract}
Under the standard assumptions, the formula for integration by parts is obtained by a straightforward calculation.
\end{abstract}

1980 Mathematics subject classification (Amer. Math. Soc.): 26 A 39

For the generalized Riemann-Stieltjes integral (abbreviated as GRS-integral) two theorems on integration by parts are presented in [3]. Unfortunately none of them is of the type one would hope for. The assumptions of the first theorem (page 115) are too strict for applications. The second theorem (page 119) is concerned with absolutely integrable functions only - a restriction alien to the spirit of the GRS-integral.

The purpose of this note is to show that with a minimal effort one can generalize the theorem of [3], Section 7.2, page 115 to its full natural strength. To keep the exposition reasonably self-contained, first we shall recall the definition of the GRS-integral. For the basic properties of the integral, we refer to [3], Chapter 7 .

By $\mathbf{R}$ and $\mathbf{R}_{+}$we denote the sets of all real and all positive real numbers, respectively. Throughout, let $I=[a, b]$ be a compact interval, and let $\alpha: I \rightarrow \mathbf{R}$. A partition of $I$ is a set $P=\left\{x_{0}, \ldots, x_{p} ; \xi_{1}, \ldots, \xi_{p}\right\}$ of real numbers such that $a=x_{0}<x_{1}<\cdots<x_{p}=b$ and $x_{i-1} \leqslant \xi_{i} \leqslant x_{i}, i=1, \ldots, p$. Given $\delta: I \rightarrow \mathbf{R}_{+}$, we say that $P$ is $\delta$-fine whenever $x_{i}-x_{i-1}<\delta\left(\xi_{i}\right), i=1, \ldots, p$.

c Copyright Australian Mathematical Society 1983 
Definition. The integral over $I$ of a function $f: I \rightarrow \mathbf{R}$ with respect to $\alpha$ is the real number $\int_{a}^{b} f d \alpha$ (if it exists) satisfying the following condition: given $\varepsilon>0$, there is a $\delta: I \rightarrow \mathbf{R}_{+}$such that

$$
\left|\sum_{i=1}^{p} f\left(\xi_{i}\right)\left[\alpha\left(x_{i}\right)-\alpha\left(x_{i-1}\right)\right]-\int_{a}^{b} f d \alpha\right|<\varepsilon
$$

for each $\delta$-fine partition $\left\{x_{0}, \ldots, x_{p} ; \xi_{1}, \ldots, \xi_{p}\right\}$ of $I$.

We note that if $\int_{a}^{b} f d \alpha$ exists and $a \leqslant c \leqslant d \leqslant b$, then so does $\int_{c}^{d} f d \alpha$ (see [3], Section 2.3, page 51).

Let $f, g: I \rightarrow \mathbf{R}$ be such that $\int_{a}^{b} f d \alpha$ and $\int_{a}^{b} f d \alpha$ exist. For each $x \in I$, set

$$
F(x)=\int_{a}^{x} f d \alpha \text { and } G(x)=\int_{a}^{x} g d \alpha .
$$

By [3], Section 7.3, page 188, the functions $F, G: I \rightarrow \mathbf{R}$ are continuous at $x \in I$ wherever $\alpha$ is continuous at $x$ or $f(x)=g(x)=0$.

Theorem. Let $\alpha$ have a finite variation in $I$, and let $F$ and $G$ be continuous in $I$. Then $\int_{a}^{b}(f G+F g) d \alpha$ exists, and

$$
\int_{a}^{b}(f G+F g) d \alpha=F(b) G(b)
$$

Proof. Let $M \in \mathbf{R}_{+}$be larger than $|F(x)|$ and $|G(x)|$ for each $x \in I$, and also larger than the variation of $\alpha$ in $I$. Given $\varepsilon>0$, choose a $\delta: I \rightarrow \mathbf{R}_{+}$so that

$$
|f(\xi)| \cdot|G(\xi)-G(x)| \leqslant \frac{\varepsilon}{4 M}, \quad|g(\xi)| \cdot|F(\xi)-F(x)|<\frac{\varepsilon}{4 M}
$$

for each $\xi, x \in I$ for which $|\xi-x|<\delta(\xi)$, and that

$$
\begin{aligned}
& \sum_{i=1}^{p}\left|f\left(\xi_{i}\right)\left[\alpha\left(x_{i}\right)-\alpha\left(x_{i-1}\right)\right]-\left[F\left(x_{i}\right)-F\left(x_{i-1}\right)\right]\right|<\frac{\varepsilon}{4 M}, \\
& \sum_{i=1}^{p}\left|g\left(\xi_{i}\right)\left[\alpha\left(x_{i}\right)-\alpha\left(x_{i-1}\right)\right]-\left[G\left(x_{i}\right)-G\left(x_{i-1}\right)\right]\right|<\frac{\varepsilon}{4 M}
\end{aligned}
$$

for each $\delta$-fine partition $P=\left\{x_{0}, \ldots, x_{p} ; \xi_{1}, \ldots, \xi_{p}\right\}$ of $I$ (see the Henstock lemma in [3], Section 7.2, page 186). For such a partition $P$ we have 


$$
\begin{aligned}
\mid \sum_{i=1}^{p}[ & \left.f\left(\xi_{i}\right) G\left(\xi_{i}\right)+F\left(\xi_{i}\right) g\left(\xi_{i}\right)\right] \cdot\left[\alpha\left(x_{i}\right)-\alpha\left(x_{i-1}\right)\right]-F(b) G(b) \mid \\
= & \mid \sum_{i=1}^{p}\left\{f\left(\xi_{i}\right) G\left(\xi_{i}\right)\left[\alpha\left(x_{i}\right)-\alpha\left(x_{i-1}\right)\right]+F\left(\xi_{i}\right) g\left(\xi_{i}\right)\left[\alpha\left(x_{i}\right)-\alpha\left(x_{i-1}\right)\right]\right. \\
& \left.\quad \sum_{i=1}^{p} \mid f\left(\xi_{i}\right) G\left(\xi_{i}\right)\left[\alpha\left(x_{i}\right)-\alpha\left(x_{i-1}\right)\right]-G\left(x_{i}\right)\left[G\left(x_{i}\right)-F\left(x_{i-1}\right) G\left(x_{i-1}\right)\right]\right\} \mid \\
& \left.+\sum_{i=1}^{p} \mid F\left(\xi_{i}\right) g\left(\xi_{i}\right)\left[\alpha\left(x_{i}\right)-\alpha\left(x_{i-1}\right)\right]-F\left(x_{i-1}\right)\left[G\left(x_{i}\right)-x_{i-1}\right)\right] \\
\leqslant & \sum_{i=1}^{p}\left|f\left(\xi_{i}\right)\right| \cdot\left|G\left(\xi_{i}\right)-G\left(x_{i}\right)\right| \cdot\left|\alpha\left(x_{i-1}\right)-\alpha\left(x_{i}\right)\right| \\
& +\sum_{i=1}^{p}\left|G\left(x_{i}\right)\right| \cdot\left|f\left(\xi_{i}\right)\left[\alpha\left(x_{i}\right)-\alpha\left(x_{i-1}\right)\right]-\left[F\left(x_{i}\right)-F\left(x_{i-1}\right)\right]\right| \\
& +\sum_{i=1}^{p}\left|g\left(\xi_{i}\right)\right| \cdot\left|F\left(\xi_{i}\right)-F\left(x_{i-1}\right)\right| \cdot\left|\alpha\left(x_{i}\right)-\alpha\left(x_{i-1}\right)\right| \\
& +\sum_{i=1}^{p}\left|F\left(x_{i-1}\right)\right| \cdot\left|g\left(\xi_{i}\right)\left[\alpha\left(x_{i}\right)-\alpha\left(x_{i-1}\right)\right]-\left[G\left(x_{i}\right)-G\left(x_{i-1}\right)\right]\right| \\
& +\varepsilon
\end{aligned}
$$

and the theorem is proved.

Using [3], Section 7.2, page 186, we obtain the corollary which properly generalizes the observation from page $195 \mathrm{ibid}$.

COROLlary. Let $\alpha$ have a finite variation in $I$, and let $F$ and $G$ be continuous in $I$. If $\int_{a}^{b} F d G$, exists, then so does $\int_{a}^{b} G d F$, and

$$
\int_{a}^{b} G d F=F(b) G(b)-\int_{a}^{b} F d G .
$$

While Example 1 below indicates that the Corollary is about as general as one can go, in some cases the next simple proposition is easier to apply. 
Proposition. Let $F$ be continuous in $I$, and let $H: I \rightarrow \mathbf{R}$ have a finite variation in I. Then $\int_{a}^{b} F d H$ and $\int_{a}^{b} f H d \alpha$ exist, and

$$
\int_{a}^{b} f H d \alpha=F(b) H(b)-\int_{a}^{b} F d H .
$$

Proof (suggested by R. Vyborny). Since the integral $\int_{a}^{b} F d H$ exists as the usual Stieltjes integral, so does $\int_{a}^{b} H d F$, and

$$
\int_{a}^{b} H d F=F(b) H(b)-\int_{a}^{b} F d H
$$

(see [3], Sections 7.5 and 7.6, pages 194 and 196). The proposition follows from [3], Section 7.2, page 186 .

In the following two examples, we shall assume that $I=[0,1]$, and $\alpha(x)=x$ for each $x \in I$. We shall write $\int_{0}^{1} f$ instead of $\int_{0}^{1} f d \alpha$.

Example 1. This example is taken from [2], Part 11, Exercise 13. For $x \in(0,1]$, let

$$
F(x)=x^{2} \sin x^{-4}, \quad G(x)=x^{2} \cos x^{-4},
$$

and set $F(0)=G(0)=0$. Then the finite derivatives $F^{\prime}=f$ and $G^{\prime}=g$ exist everywhere in $I$, yet the integrals

$$
\int_{0}^{1} F g=\int_{0}^{1} F d G \text { and } \int_{0}^{1} f G=\int_{0}^{1} G d F
$$

do not exist. Thus even in a very simple situation, the assumptions of the Theorem do not guarantee the existence of $\int_{a}^{b} F d G$ or $\int_{a}^{b} G d F$.

EXAMPLE 2. For $x \in(0,1]$, let

$$
F(x)=x^{2} \sin x^{-2}, \quad g(x)=x^{-1} \sin x^{-1},
$$

and set $F(0)=g(0)=0$. Then the finite derivative $F^{\prime}=f$ exists everywhere in $I$, and also $\int_{0}^{1} g$ exists. By the Corollary, $\int_{0}^{1} f G=\int_{0}^{1} G d F$ exists, and

$$
\int_{0}^{1} f G=F(b) G(b)-\int_{0}^{1} F d G
$$

for the continuity of $\mathrm{Fg}$ implies the existence of $\int_{0}^{1} F g=\int_{0}^{1} F d G$.

This result cannot be obtained from the Proposition. Indeed, since neither $\int_{0}^{1}|f|$ not $\int_{0}^{1}|g|$ exists, by [3], Section 3.2, both $F$ and $G$ are of infinite variation in $I$. The converse situation, in which the Corollary cannot be applied while the Proposition can, occurs typically when $H: I \rightarrow \mathbf{R}$ is a function of finite variation which is singular with respect to $\alpha$. 
REMARK. Suppose that $\alpha$ is increasing in $I$. Then it is not hard to show (see [4], Corollary B5 and Remark B13) that the GRS-integral coincides with the PerronStieltjes integral defined in [2], Part II, Sections 41, 47, and 88. For the Perron-Stieltjes integral, a rather complicated proof of the Theorem can be found in [2], Part II, Exercises 20-23. The proposition was originally proved for the Perron integral by means of the deep Hake-Alexandrov-Looman theorem which identifies the Perron and Denjoy integrals (see [5], Chapter VIII, Sections 2 and 3 ). Elementary, but laborious proofs of the proposition based directly on the Perron definition were given in [2], Part II, Section 92-94 and [1]. In comparison with these proofs the simplicity of our presentation is another strong argument in favour of the GRS-integral.

This paper was prepared while the author visited the Royal Institute of Technology in Stockham and the Monash University in Melbourne.

\section{References}

[1] L. Gordon and S. Lasher. 'An elementary proof of integration by parts for the Perron integral', Proc. Amer. Math. Soc. 18 (1967), 394-398.

[2] J. Mařik, 'Foundation of the theory of an integral in Euclidean spaces', Časopis Pěst. Mat. 77 (1952), 1-51, 125-144, 267-30l (translated to English by L. I. Trudzik, Dept. of Math., Univ. of Melbourne, Parkville, Victoria 3052, Australia).

[3] R. M. McLeod, The generalized Riemann integral (MAA, Providence, 1980).

[4] W. F. Pfeffer, 'The Riemann-Stieltjes approach to integration', TWISK 187, CSIR, Pretoria, 1980.

[5] S. Saks, Theory of the integral (Hafner, New York, 1939).

Department of Mathematics

University of California

Davis, California 95616

U.S.A. 